\title{
Plateau Modulus of Epoxidized Polybutadiene
}

\author{
C. M. Roland* \\ Chemistry Division, Code 6120, Naval Research Laboratory, Washington, D.C. 20375-5342
}

\author{
J. K. Kallitsis and K. G. Gravalos
}

Department of Chemistry, University of Patras, 26500 Patras, Greece

Received June 21, 1993; Revised Manuscript Received August 23, $1993^{\circ}$

\begin{abstract}
Dynamic mechanical spectra in the terminal and plateau regions were measured for 1,4-polybutadiene (PBD) epoxidized to different degrees. The chemical modification results in a chain of increased flexibility and larger cross-sectional area. Although small, both structural changes are anticipated from current theories to contribute to reduced topological interaction among chains. Notwithstanding, the plateau modulus was observed to systematically increase with epoxidation level. The results are similar to those found previously for epoxidized polyisoprene, ${ }^{1}$ in which the effect of chemical structure on chain entanglements was also contrary to expectations. This qualitative failure of available predictions suggests that the relationship between chemical structure and entanglement interactions remains an open question.
\end{abstract}

\section{Introduction}

The plateau region in the viscoelastic spectrum is of particular interest because it reflects a distinguishing characteristic of polymeric materials; viz., they are comprised of long, flexible, and uncrossable molecules. The latter feature suppresses long-range reconfigurations, giving rise to the elastic response of the rubbery plateau. The magnitude of the plateau modulus, $G_{0}$, is governed by the concentration and effectiveness of the topological constraints. The physical origin of these entanglements and their dependence on chemical structure have been addressed by many investigators. Scaling arguments are commonly employed, based generally on the idea of Graessley and Edwards ${ }^{2}$ that the chain contour length per unit volume governs the magnitude of the topological constraints. However, since there are two relevant length scales, the interchain distance and the Kuhn step length, scaling approaches alone cannot yield a unique solution to the problem. 3,4 Some idea concerning the nature of an entanglement must be introduced, leading to some quantitative relationship between chemical structure and the plateau modulus. Various definitions of an entanglement coupling have been proposed, including a fixed number of binary contacts per entanglement, ${ }^{5,6}$ a fixed number of binary contacts per entanglement volume, ${ }^{7,8}$ and a fixed number of strands per entanglement volume. ${ }^{9-11}$ Although differing quantitatively, these approaches ${ }^{2,5-11}$ have in common the prediction that increases in chain flexibility and in chain bulkiness will reduce the plateau modulus. It is certainly plausible that either factor would diminish topological encounters with neighboring chains; more flexible chains pervade less of the surrounding volume, and the backbone of chains with large pendant groups is more removed from neighboring chains.

An alternative to scaling approaches, developed by Iwata and Edwards, ${ }^{12,13}$ is based on calculation of topological invariants. The entanglement capability of a polymer is expressed in terms of a topological interaction parameter, which can in turn be related to the plateau modulus and to the second virial coefficient. In accord with scaling theories, a conclusion drawn from the topological calculations is that macromolecules having larger diameters or greater flexibility (i.e., a smaller characteristic ratio, $C_{\infty}$ ) are less entangled. ${ }^{12}$

Abstract published in Advance ACS Abstracts, October 15, 1993.
In this paper we describe the effect of epoxidation on the plateau modulus of 1,4-polybutadiene. The conversion of the main-chain unsaturated carbons to oxirane moieties (to form random copolymers of 1,2-epoxy-1,4-butanediyl and butenylene) alters the chemical structure in a manner expected to influence the entanglement propensity of the polymer. The epoxy moiety projects roughly $1.2 \AA$ from the chain backbone, ${ }^{14}$ thus effecting a modest increase in the chain cross-sectional area. In addition, the oxirane ring has some torsional compliance, ${ }^{14}$ and thus more flexibility than the precursor vinyl carbons. Intrinsic viscosity measurements corroborate the increased chain flexibility of epoxidized polydienes. ${ }^{1}$ These increases in both the bulkiness and flexibility should, according to presently available theories, result in a polymer with reduced entanglement couplings, and hence a lower plateau modulus.

\section{Experimental Section}

The linear polybutadiene ( $49 \%$ trans- $1,4,41 \%$ cis- 1,4 , and $10 \%$ 1,2 chain units) was obtained from Polymer Labs and had a molecular weight equal to 120000 with a polydispersity of 1.03 . The polybutadiene was epoxidized to various levels using peracetic acid, as described previously. ${ }^{15}$ The epoxidation reaction is random, specific for the 1,4 chain units, and maintains the stereochemistry. ${ }^{16}$ The degree of epoxidation of the modified polymers, as determined by proton NMR, is given in Table I.

The glass transition temperatures (Table I) were obtained using a Perkin-Elmer DSC-2 at a $10^{\circ} \mathrm{C} / \mathrm{min}$ scan rate. Dynamic mechanical data in the plateau and terminal zones were obtained with a Bohlin VOR rheometer using a parallel-plate geometry. Sample diameters of 15,25 , and $40 \mathrm{~mm}$ were used, with gaps in the range of 0.6-1.7 mm. Each sample was measured using at least two different diameter plates. The shear modulus was measured typically from $10 \mathrm{~Hz}$ down to as low as $2 \times 10^{-4} \mathrm{~Hz}$ at temperatures between -25 and $+45^{\circ} \mathrm{C}$. Temperature control was $\pm 0.1^{\circ} \mathrm{C}$ or better.

\section{Results}

In Figures 1 and 2 are displayed the storage, $G^{\prime}(\omega)$, and the loss, $G^{\prime \prime}(\omega)$, modulus master curves for the four polymers. Superpositioning of the data required modest vertical shifting; the measured moduli were a weakly decreasing function of temperature, in agreement with previous results for polybutadiene ${ }^{17}$ and polyisoprene. ${ }^{18}$ The empirically determined horizontal (time-temperature) shift factors, expressing the ratio of the relaxation 
Table I

\begin{tabular}{lccccc}
\hline polymer & epoxide, mol $\%$ & $T_{\mathrm{g}}{ }^{\circ} \mathrm{C}$ & $c_{1}{ }^{a}$ & $c_{2},{ }^{a}$ deg & $G_{0}, \mathrm{MPa}$ \\
\hline PBD & 0 & -99.5 & $\mathbf{2 . 9 5 4}$ & 158.1 & 1.31 \\
EPBD-35 & 35.4 & -60.5 & 3.643 & 128.2 & 1.49 \\
EPBD-41 & 40.5 & -50.4 & 3.940 & 122.2 & 1.51 \\
EPBD-58 & 58.0 & -43.9 & 6.905 & 180.0 & 1.60
\end{tabular}

a Equation 1 with $T_{0}=303 \mathrm{~K}$.

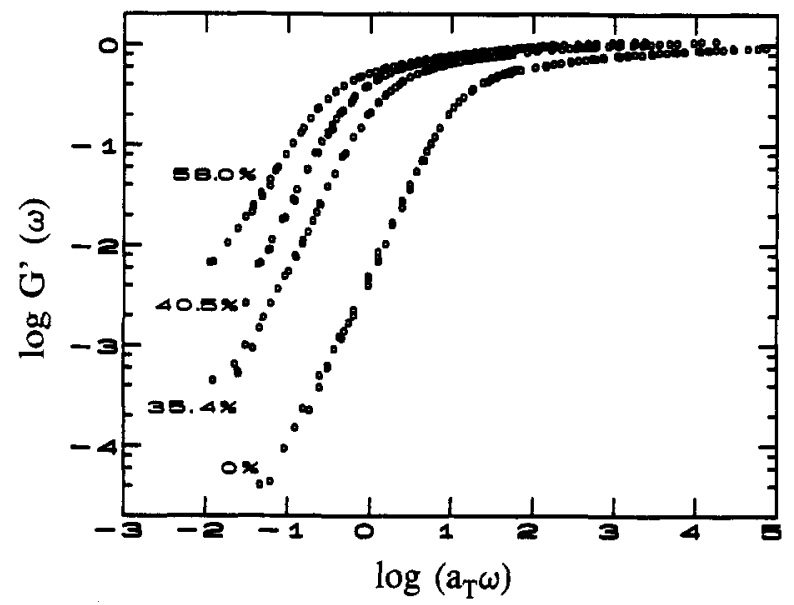

Figure 1. Logarithmic plots of the storage modulus master curves for 1,4-polybutadiene with the indicated level of epoxidation. The reference temperature is in all cases $30^{\circ} \mathrm{C}$.

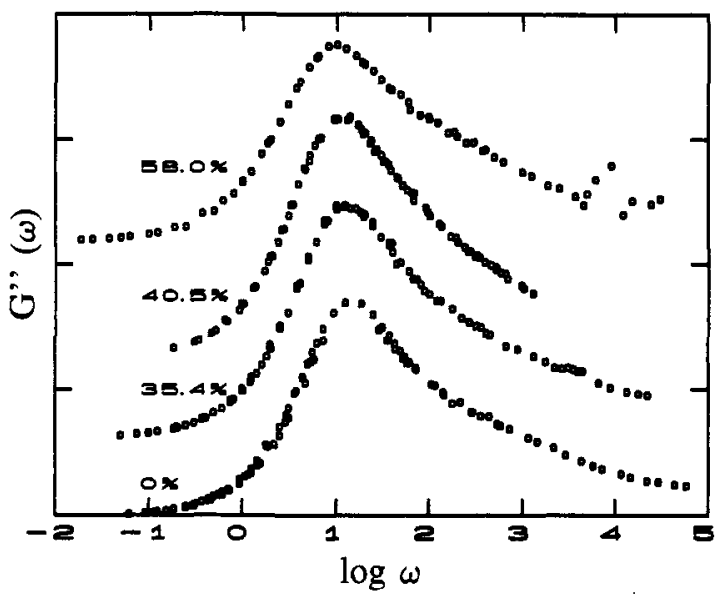

Figure 2. Semilogarithmic plots of the terminal dispersion in the loss modulus for polybutadiene epoxidized to various degrees. The curves have been arbitrarily shifted for clarity.

time at a given temperature, $T$, to that at a reference temperature, $T_{0}$, conformed to the WLF equation, ${ }^{19}$

$$
\log a_{T}=\frac{-c_{1}\left(T-T_{0}\right)}{c_{2}+T-T_{0}}
$$

The WLF constants, listed in Table I, vary with epoxidation level; however, as seen in Figure 3 the terminal relaxation times, defined to be $\left(2 \pi f_{\max }\right)^{-1}$ where $f_{\max }$ is the frequency of the maximum of the loss modulus peak, have a similar temperature dependence. In fact, the commonly employed temperature variable $T-T_{\mathrm{g}}$ suffices to roughly superpose the data for the different polymers.

The value of the storage modulus in the plateau region of Figure 1 is determined by the magnitude of the entanglement constraints. Due to the relatively low molecular weight of the polymers, however, a strict invariance to frequency is not observed; consequently, $G^{\prime}(\omega)$ cannot be used to quantify the effect of epoxidation on entanglements. Application of the Kronig-Kramers relation to the terminal dispersion in $G^{\prime \prime}(\omega)$ yields a value

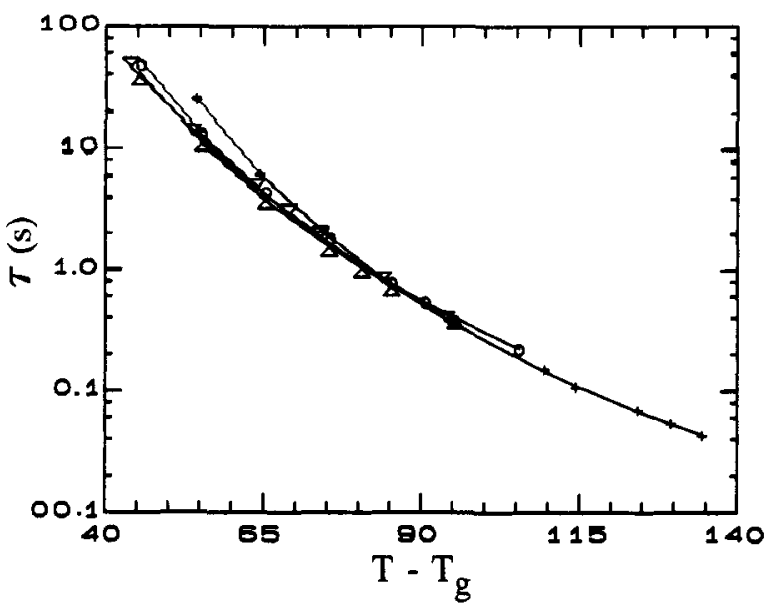

Figure 3. Terminal relaxation time determined for polybutadiene with $0(+), 35.4(0), 40.5(\Delta)$, and $58.0(\nabla) \mathrm{mol} \%$ epoxidation, as a function of the temperature above the glass transition temperature. The curves through the data points correspond to the best fit to the WLF equation. The WLF constants and glass transition temperatures are given in Table I.

for the modulus of the entanglement network ${ }^{19,20}$

$$
G_{0}=\frac{2}{\pi} \int_{\mathrm{a}}^{\mathrm{b}} G^{\prime \prime}(\omega) \mathrm{d} \ln \omega
$$

The contribution from the glass transition region must be excluded from the integral, introducing a small error into the value obtained for $G_{0}$. Many functional forms for the terminal dispersion are available, ${ }^{21}$ and these can provide a means to extrapolate the high-frequency side of the dispersion. We are interested herein in the change in $G_{0}$ upon epoxidation. A straightforward method to obtain $G_{0}$ is to numerically integrate the low-frequency side and then fit the high-frequency data to a power law. ${ }^{22}$ The latter is then integrated analytically to complete the calculation of eq 2 .

The results for $G_{0}$ using the power law extrapolation are given in Table I. The plateau modulus determined for the unmodified polymer, $1.3 \mathrm{MPa}$, is within the range of values reported previously for polybutadienes of similar microstructure, $1.1 \leq G_{0} \leq 1.3 \mathrm{MPa}{ }^{22-25}$ Epoxidation systematically increases the plateau modulus (Table I). Although the changes are not large, they are reproducible and significant.

Different procedures for calculating eq 2 were also tested. The dispersion could be well-fitted on the low-frequency side to the stretched exponential function, ${ }^{26,27}$ with the fitted function then integrated analytically. This procedure gave somewhat lower values for $G_{0}$ than those in Table I; however, the relative magnitudes of $G_{0}$ for the four polymers were quite similar for the two methods. For example, at $58 \mathrm{~mol} \%$ epoxidation, the increase in $G_{0}$ was determined to be $22 \%$ (Table I) using numerical integration and a power law to extrapolate the high-frequency side of the dispersion, while an $18 \%$ increase was calculated by integrating a fitted stretched exponential function.

\section{Discussion}

The projection of the oxirane ring from the epoxidized polybutadiene backbone increases the average transverse chain dimension. Epoxidation of the vinyl carbons also introduces some torsional compliance, resulting in a smaller characteristic ratio. (This is reflected, for example, in the $32 \%$ smaller intrinsic viscosity of $50 \%$ epoxidized polyisoprene, when measured in THF, a better solvent for 


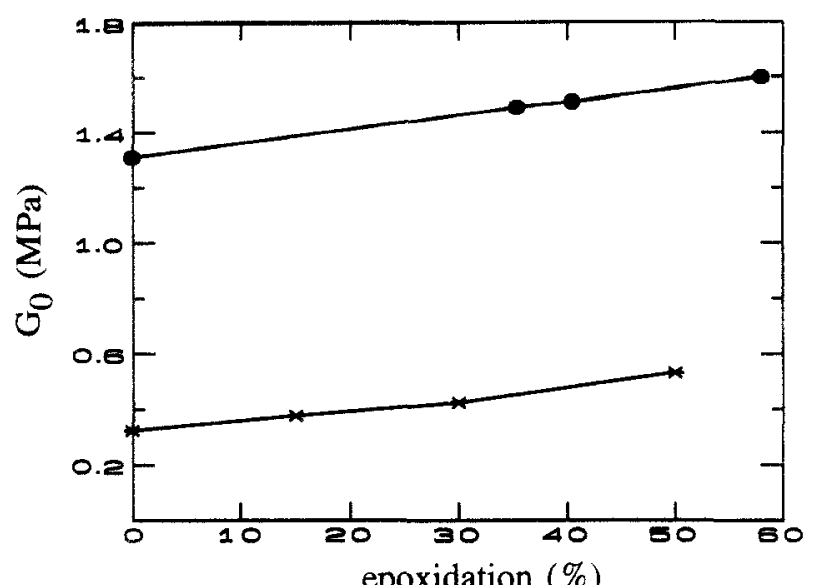

epoxidation (\%)

Figure 4. Dependence of the plateau modulus on the extent of epoxidation for 1,4-polybutadiene (๑) and cis-1,4-polyisoprene (X).

the modified polymer. ${ }^{1}$ ) Both structural changes are expected to diminish the plateau modulus. Although the magnitude of the effect depends on the particular definition of an entanglement coupling employed, ${ }^{8}$ all scaling theories, as well as the topological model, ${ }^{12,13}$ predict that flexibility and chain bulkiness reduce the entanglement density. In the present work, as well as in an earlier study of epoxidized polyisoprene, these predictions are found to be qualitatively in error - the epoxidized polydienes have larger plateau moduli (Figure 4).

There have been many empirical attempts to correlate the magnitude of the plateau modulus with chemical structure. ${ }^{28-32}$ In part reflecting experimental errors and uncertainties concerning the values of the required molecular parameters, these correlations at best provide a general trend about which compilations of measured $G_{0}$ exhibit large scatter. Thus, assessment of the validity of theoretical predictions must rely on judging the quality of the agreement with experimental data; any conclusions are equivocal.

The value of the present results and of an earlier study of polyisoprene ${ }^{1}$ is that the changes in structural characteristics occasioned by the chemical modification are expected to uniformly tend in the same direction; that is, toward reduction in the plateau modulus. No problems arise herein from inaccuracies in the molecular parameters (e.g., Kuhn step length, chain density, etc.) required to calculate a theoretical value for $G_{0}$, since the present data are in qualitative disagreement with theory. The fact that the experimental results are in the opposite direction from predictions clearly demonstrates the need for a better understanding of the entanglement phenomenon. The larger plateau modulus in the more flexible epoxidized polymers is congruent with the idea of entanglements as hooks or knots, ${ }^{33}$ greater flexibility facilitating such interchain "hooking". In fact, the theory of $W^{34,35}$ predicts that more flexible chains are more entangled. Although models of this type have received less attention than scaling approaches to the chain entanglement problem, such ideas may deserve more serious consideration.
A recent study 4 explored the effect on entanglements of systematic changes in the two length scales relevant to topological constraints, the interchain distance and the Kuhn step length. The results were found to be quantitatively at odds with scaling predictions. One conclusion of this study was that purely geometrical models are insufficient to describe all experimental data; dynamic contributions to the plateau modulus must be considered.

Acknowledgment. The authors are grateful to D. W. Tomlin and K. J. McGrath for carrying out DSC and NMR measurements, respectively. The work at NRL was supported by the Office of Naval Research.

\section{References and Notes}

(1) Roland, C. M. Macromolecules 1992, 25, 7031.

(2) Graessley, W. W.; Edwards, S. F. Polymer 1981, 22, 1329.

(3) Helfand, E. In Photophysical and Photochemical Tools in Polymer Science; Winnik, M. A., Ed.; Reidel: New York, 1986.

(4) Richter, D.; Farago, B.; Butera, R.; Fetters, L. J. Juang, J. S.; Ewen, B. Macromolecules 1993, 26, 795.

(5) Edwards, S. Proc. Phys. Soc. 1967, 92, 9

(6) Brochard, F.; De Gennes, P.-G. Macromolecules 1977, 10, 1157.

(7) Colby, R. H.; Rubinstein, M. Macromolecules 1990, 23, 2753.

(8) Colby, R. H.; Rubinstein, M. Viovy, J.L. Macromolecules 1992, $25,996$.

(9) Ronca, G. J. Chem. Phys. 1983, 79, 1031.

(10) Lin, Y.-H. Macromolecules 1987, 20, 3080.

(11) Kavassalis, T. A.; Noolandi, J. Macromolecules 1988, 21, 2869; $1989,22,2709$.

(12) Iwata, K.; Edwards, S. F. J. Chem. Phys. 1989, 90, 4567.

(13) Iwata, K.; Edwards, S. F. Macromolecules 1988, 21, 2901.

(14) Rosowsky, A. In Heterocyclic Compounds with Three-and FourMembered Rings; Weissberger, A., Eds.; Interscience: New York, 1964.

(15) Gravalos, K. G.; Kalfoglou, N. K. J. Appl. Polym. Sci. 1992, 45, 2731.

(16) Gelling, 1. R.; Porter, M. In Natural Rubber Science and Technology; Roberts, A. D., Ed.; Oxford University Press: Oxford, U.K., 1988; p 359.

(17) Carella, J. M.; Graessley, W. W.; Fetters, L. J. Macromolecules $1984,17,2775$.

(18) Gotro, J. T.; Graessley, W. W. Macromolecules 1984, 17, 2767.

(19) Ferry, J. D. Viscoelastic Properties of Polymers; Wiley: New York, 1980.

(20) Sanders, J. F.; Ferry, J. D. Macromolecules 1969, 2, 440.

(21) Wasserman, S. H.; Graessley, W. W. J. Rheol. 1992, 36, 543.

(22) Colby, R. H.; Fetters, L. J.; Funk, W. G.; Graessley, W. W. Macromolecules 1991, 24, 3873.

(23) Raju, V. R.; Menezes, E. V.; Marin, G.; Graessley, W. W. Macromolecules 1981, 14, 1668.

(24) Struglinski, M. J.; Graessley, W. W. Macromolecules 1985, 18, 2630 .

(25) Mancke, R. G.; Dickie, R. A.; Ferry, J. D. J. Polym. Sci., Polym. Phys. Ed. 1968, 6, 1783 .

(26) Ngai, K. L.; Rendell, R. W.; Rajagopal, A. K.; Teitler, S. Ann. N.Y. Acad. Sci. 1986, 484, 150 .

(27) Ngai, K. L.; Plazek, D. J. J. Polym. Sci., Polym. Phys. Ed. 1985, $23,2159$.

(28) Fox, T. G.; Allen, V. R. J. Chem. Phys. 1964, 41, 344.

(29) van Krevelen, D. W. Properties of Polymers, 2nd ed.; Elsevier: Amsterdam, The Netherlands, 1976.

(30) Boyer, R. F.; Miller, R. L. Rubber Chem. Technol. 1978, 51, 718.

(31) Privalko, V. P. Macromolecules 1980, 13, 370.

(32) Aharoni, S. M. Macromolecules 1983, 16, 1722; 1986, 19, 426.

(33) Reference 19, Figures 10-12 (II), p 245.

(34) Wu, S. J. Polym. Sci. 1989, 27, 723.

(35) Wu, S. Polym. Eng. Sci. 1993, 33, 289. 